\title{
$H-T$ PHASE DIAGRAM OF MAGNETIC SEMICONDUCTOR THIN FILMS - EXPERIMENT
}

\author{
M. LUBecka ${ }^{a}$, L.J. MaKsymowicz ${ }^{a}$, R. SzYMCZak ${ }^{b}$ AND W. PowroźNiK ${ }^{a}$ \\ ${ }^{a}$ Department of Electronics, UM \& M, Al. Mickiewicza 30, 30-059 Kraków, Poland \\ bInstitute of Physics, Polish Academy of Sciences
}

Al. Lotników 32/46, 02-668 Warszawa, Poland

We used the irreversibilities between the field cooled and zero field cooled dc magnetization to determine the field and composition dependence of the spin-glass freezing temperature in $\mathrm{CdCr}_{2} \mathrm{Se}_{4}:$ In (REE) and $\mathrm{CdCr}_{2-2 x} \operatorname{In}_{2 x} \mathrm{Se}_{4}(0.15 \leq x \leq 0.35)$ (SG) thin films. The $H-T$ phase diagram of samples with REE has two instability lines: the Gabay-Toulouse-type (G-T) and the De Almeida-Thouless-type (A-T) while samples in SG state are characterized by the A-T line. The A-T line of thin films was used for calculation the normalised internal magnetic field $h_{\mathrm{m}}$ of infinite spin clusters with long range ordering.

PACS numbers: $64.60 . \mathrm{Cn}, 75.50 . \mathrm{Lk}, 75.30 . \mathrm{Gw}$

Chalcogenide spinels of $\mathrm{CdCr}_{2} \mathrm{Se}_{4}$ exhibit specific properties that can be controlled by the dilution level. When the concentration of magnetic atoms is clianged, the following magnetic phases are obtained:

(i) for $\mathrm{CdCr}_{2} \mathrm{Se}_{4}: \mathrm{In}$, the ferromagnetic state (FM) with the reentrant transition (REE). The system in microscopic scale is considered as consisting of an infinite ferromagnetic network (IFN) with long range ferromagnetic order and finite spin clusters (FSC) randomly distributed in IFN. The amount of IFN significantly dominates the FSC, in the volume of sample. The system has two instability lines at the $H-T$ phase diagram: the De Almeida-Thouless (A-T) line [1], which indicates the onset of longitudinal freezing and Gabay-Toulouse $(G-T)$ line for the transverse freezing [2].

(ii) for $\mathrm{CdCr}_{2-2 x} \mathrm{In}_{2 x} \mathrm{Se}_{4}(0 \ll x \leq 0.4)$, spin-glass state. With increasing amount of indium the FSC dominates over IFN. This system exhibits the A-T instability line. This microscopic picture of both REE and SG develops the problem of the instability A-T and G-T lines [1-4]. Introducing the IFN, the internal magnetic field, related to IFN, has to be taken under consideration in the description of instability lines.

The disordered magnetic system is macroscopically characterised by: 
(i) the temperature dependence of induced magnetization $M$, which includes the non-zero density of states in the energy gap [5],

(ii) the unidirectional magnetic anisotropy field $H_{\text {an }}$ originated from the Dzaloshinsky-Moriya interaction (D-M).

Thin films of $\mathrm{CdCr}_{2-2 x} \operatorname{In}_{2 x} \mathrm{Se}_{4}$ (for $0 \leq x \leq 0.4$ ) obtained by rf sputtering were investigated in the temperature range from $4 \mathrm{~K}$ to $200 \mathrm{~K}$. The basic parameters $M(T)$ and $H_{\text {an }}(T)$, which classify the magnetic phase of samples, were determined from ferromagnetic resonance (FMR) [5] and SQUID data. The $H_{\text {an }}$ was also determined from the displacement of $M-H$ loop. The hysteresis loops were measured after cooling the samples from $120 \mathrm{~K}$ to $4.2 \mathrm{~K}$ in the external magnetic field $H_{F C}$. The magnetic anisotropy induced by the field cooling is unidirectional with a single easy direction of magnetization parallel to $H_{\mathrm{FC}}[6]$. For a chosen value of $H_{\mathbf{F C}}$ several cycles of loops were recorded, each loop up to different external magnetic field $H_{\max }$. Asymmetrical loops were obtained until $H_{\max }$ has reached the value of $H_{\text {an }}$ that was ascribed to the unidirectional magnetic anisotropy. A further increase in $H_{\max }$ produces the reversible, symmetrical $M-H$ loops. Figure 1 presents the two cycles of $M-H$ loops for thin films of $\mathrm{CdCr}_{1.3} \mathrm{In}_{0.7} \mathrm{Se}_{4}$. The $M-H$ loop is asymmetrical for $H_{\max } \leq 300$ Oe it means that $H_{\mathrm{a}} \mathrm{n}=300 \mathrm{Oe}$. The freezing lines were found from the onset of irreversibilities between the field cooled (FC) and zero field cooled (ZFC) dc magnetization. We analysed the phase diagram $\left(H_{\mathrm{a}}-T\right)$ in terms of De Almeida-Thouless model [1] and of Gabay-Toulouse model $[2,4]$.

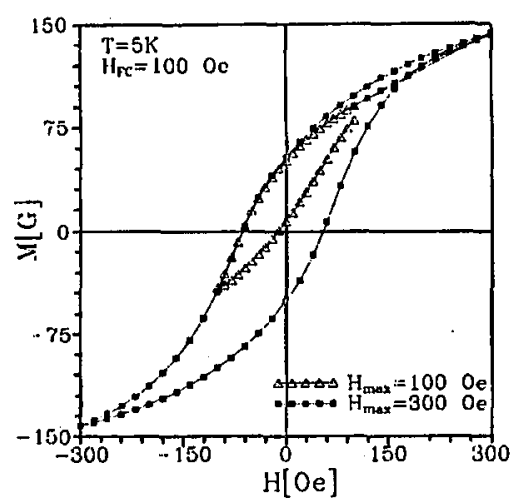

Fig. 1. $M-H$ loop for $\mathrm{CdCr}_{1.7} \operatorname{In}_{0.3} \mathrm{Se}_{4}$ thin film taken at $T=5 \mathrm{~K}, H_{\mathrm{FC}}=100 \mathrm{Oe}$.

In our investigations we used the FC and ZFC dc magnetization measurements done by SQUID. The dc magnetization $\left(M_{\mathrm{FC}}\right.$ and $\left.M_{\mathrm{ZFC}}\right)$ as a function of temperature was measured for a set of external magnetic fields $H_{\mathrm{a}}$ applied within the film plane. On the base of these experimental data, for the fixed value of $H_{\mathrm{a}}$, the irreversibility of magnetization $\left(\Delta M=M_{\mathrm{FC}}-\mathrm{M}_{\mathrm{ZFC}}\right)$ vs. temperature was obtained. Then $H_{\mathrm{a}}-T$ phase diagram was found. Depending on the samples composition the character of $\Delta M(T)$ curve exhibits one or two critical temperatures. Following the paper [7] we defined: 

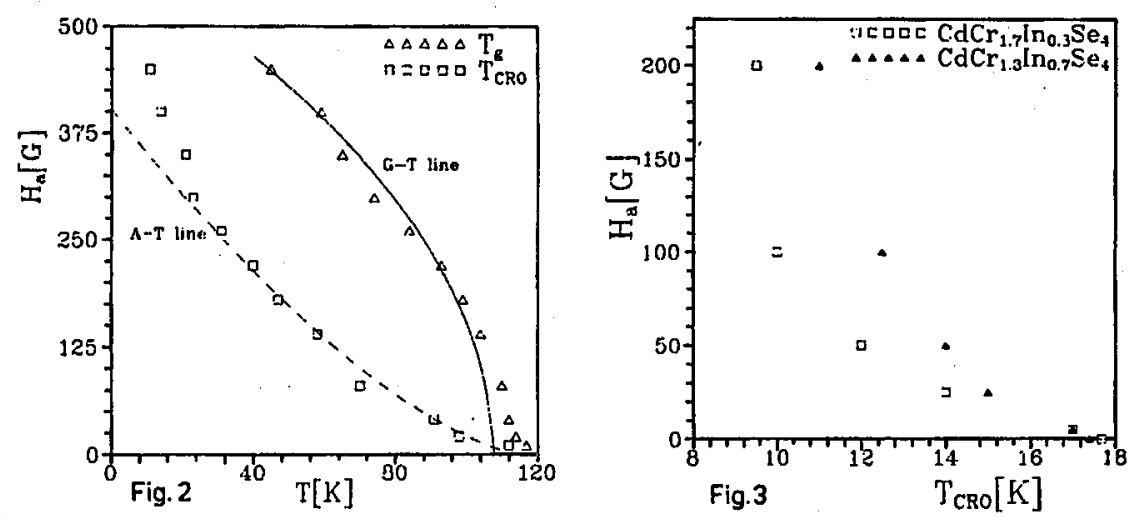

Fig. 2. $H_{\mathrm{a}}-T$ phase diagram of $\mathrm{CdCr}_{2} \mathrm{Se}_{4}$ :In thin film (REE).

Fig. 3. $H_{\mathrm{a}}-T$ phase diagram of $\mathrm{CdCr}_{1.7} \operatorname{In}_{0.3} \mathrm{Se}_{4}$ and $\mathrm{CdCr}_{1.3} \operatorname{In}_{0.7} \mathrm{Se}_{4}$ thin films (SG).

$-T_{\mathbf{g}}$ as the temperature below which the first onset of irreversibilities is seen,

- $T_{\text {CRO }}$ is the temperature below which $\Delta M$ increases relatively rapidly.

If only one critical temperature is present the A-T or G-T line can be established. When two critical temperatures are found, the phase diagram has the A-T and G-T lines [7]. Figure 2 presents the experimentally determined $H_{\mathrm{a}}-T$ curves for the thin films of $\mathrm{CdCr}_{2} \mathrm{Se}_{4}: \mathrm{In}$ (REE). It is seen that above $T=100 \mathrm{~K}$ the character of $\mathrm{G}-\mathrm{T}$ line changes to A-T line. Figure 3 shows the A-T lines for $\mathrm{CdCr}_{1.7} \mathrm{In}_{0.3} \mathrm{Se}_{4}$ and $\mathrm{CdCr}_{1.3} \mathrm{In}_{0.7} \mathrm{Se}_{4}$ thin films. We also have analysed the experimental data using the linear relationship between $h_{\text {eff }}$ and $\tau^{\alpha}$ in the form predicted by De Almeida-Thouless (A-T line):

$$
\begin{aligned}
& h_{\text {eff }}=(8 /(n+1) /(n+2))^{1 / 2} \tau_{1}^{3 / 2}, \\
& \text { where } \tau_{1}=1-T_{\mathrm{CRO}}(H) / T_{\mathrm{CRO}}(0) \text { and } n=1
\end{aligned}
$$

and in the form predicted by Gabay-Toulouse (G-T line):

$$
\begin{aligned}
& h_{\text {eff }}=2(n+2) /\left(n^{2}+4 n+2\right)^{1 / 2} \tau_{2}^{1 / 2}, \\
& \text { where } \tau_{2}=1-T_{\mathrm{g}}(H) / T_{\mathrm{g}}(0) \text { and } n=3 .
\end{aligned}
$$

The reduced field $h_{\text {eff }}=h_{\mathrm{a}}+h_{\mathrm{m}}$.

The normalized external field $h_{\mathrm{a}}=\eta g S_{\mathrm{eff}} \mu_{\mathrm{B}} H_{\mathrm{a}} / k_{\mathrm{B}} T_{g}(0)$ for G-T line and for A-T line $h_{\mathrm{a}}=\eta g S_{\text {eff }} \mu_{\mathrm{B}} H_{\mathrm{a}} / k_{\mathrm{B}} T_{\mathrm{CRO}}(0)$, with $g=2, S_{\text {eff }}=3 / 2$ for $\mathrm{Cr}^{3+}$ and $\eta=60$ (average size of clusters [7]). The internal field $h_{\mathrm{m}}$ is related to IFN, $h_{\mathrm{m}}=m J_{0}$ and $m$ is the normalized macroscopic induced magnetization, $J_{0}$ is the exchange constant of IFN. The internal magnetic field $h_{\mathrm{m}}$, calculated for each value of $h_{\mathrm{a}}$, was found to be dilution level dependent. The $h_{\mathrm{m}}$ decreases when amount of indium is increasing. The data of $\mathrm{CdCr}_{2} \mathrm{Se}_{4}: \mathrm{In}$ (REE) and $\mathrm{CdCr}_{2-2 x} \operatorname{In}_{2 x} \mathrm{Se}_{4}$ (SG) thin films are collected in Table for $\tau_{1}=\tau_{2}=0.20$. We found that the exchange constant $J_{0}$ of IFN, decreases with increasing In concentration. 


\section{TABLE}

Reduced field for different compounds.

\begin{tabular}{l|c|c|c|c}
\hline \hline \multicolumn{1}{c|}{ Sample } & $h_{\text {eff }}$ & $h_{\mathrm{a}}$ & $h_{\mathrm{m}}$ & $J_{0}=1-h_{\mathrm{a}} / h_{\text {eff }}$ \\
\hline $\mathrm{CdCr}_{2} \mathrm{Se}_{4}: \mathrm{In}$ & & & & \\
${ }^{* * *}$ & 0.100 & 0.002 & 0.097 & 0.98 \\
${ }^{* *}$ & 0.921 & 0.020 & 0.901 & 0.98 \\
\hline $\mathrm{CdCr}_{1.7} \mathrm{In}_{0.3} \mathrm{Se}_{4}^{*}$ & 0.100 & 0.012 & 0.088 & 0.88 \\
\hline $\mathrm{CdCr}_{1.3} \mathrm{In}_{0.7} \mathrm{Se}_{4}^{*}$ & 0.100 & 0.026 & 0.074 & 0.74 \\
\hline
\end{tabular}

"A-T line, ${ }^{* *} \mathrm{G}-\mathrm{T}$ line.

The work was partly supported by the Committee for Scientific Research grant No. 8 T11B 05409.

\section{References}

[1] J.R.L. De Almeida, D.J. Thouless, J. Phys. A 11, 983 (1978).

[2] M. Gabay, G. Toulouse, Phys. Rev. Lett. 47, 201 (1981).

[3] C. Pappa, J. Hammann, C. Jacobini, J. Phys. C, Solid State Phys. 17, 1303 (1984).

[4] G. Toulouse, M. Gabay, T.L. Lubensky, J.J. Vannimenus, J. Phys. Lett. 43, L109 (1982).

[5] M. Lubecka, L.J. Maksymowicz, Phys. Rev. B 48, 951 (1993).

[6] Kh.A. Zig, J.S. Kouvel, Phys. Rev. B 41, 4579 (1990).

[7] F. Lefloch, J. Hammann, M. Ocio, E. Vincent, Physica B 203, 63 (1994). 